\title{
A portable radiometric system for nuclear medicine
}

\author{
S.V. Pankin ${ }^{a, *}$, A.I. Surdo ${ }^{\mathrm{a}, \mathrm{b}}$, A.D. Krotov ${ }^{\mathrm{a}}$, M.N. Sarychev ${ }^{\mathrm{a}}$, V.V. Pankin ${ }^{\mathrm{a}, \mathrm{c}}$, A.V. Zelenin ${ }^{\mathrm{d}}$, \\ A.V. Kruzhalov ${ }^{\mathrm{a}}$ \\ ${ }^{a}$ Ural Federal University, Mira Street 19, Ekaterinburg, 620002, Russia \\ ${ }^{\mathrm{b}}$ Institute of Industrial Ecology UB RAS, Sophy Kovalevskoy Street 20, Ekaterinburg, 620137, Russia \\ ${ }^{\mathrm{c}}$ Ural State Medical University, Repina Street 3, Ekaterinburg, 620014, Russia \\ ${ }^{\mathrm{d}}$ Regional Children's Clinical Hospital, Serafimy Deryabinoy Street 32, Ekaterinburg, 620149, Russia
}

\section{A R T I C L E I N F O}

\section{Keywords:}

Nuclear medicine

Dynamic scintigraphy

Radiopharmaceutical

Phantom

\begin{abstract}
A B S T R A C T
It's shown suggested methods for diagnosing pathological processes in biological objects using a developed portable radiometric system with four gamma-ray detectors. Designed portable radiometric system was used to make 2D imaging of anatomical structures. The system can value pharmacokinetics in several points of wide area of interest at the patient body. In this study specially designed phantoms were used. Phantom models of the liver and blood circulation system were made to assay radiometric system. The construction concept and possible applications of the system in the practice of radionuclide diagnostic studies in vivo are presented.
\end{abstract}

\section{Introduction}

The main goal of radionuclide diagnostics in vivo is quantitative assessment of a radiopharmaceutical (RFC) in a biological object. In the middle of the 20th century, scintigraphy studies in vivo were conducted by means of single-detector and multi-detector radiometric systems based on scintillation counters with vacuum photomultipliers (Pankin et al., 2016). The old systems had some disadvantages: stationary placement, large size, heavy weight, high-voltage needs (more than $1000 \mathrm{~V}$ ) and lack of digital data processing capabilities (the systems were manufactured before the era of personal computers). Currently, the most common technical solution for conducting radionuclide diagnostics is the use of a gamma camera (Rosental et al., 1995). It is important to note that these technical devices also feature disadvantages that limit their functionalities. Because of its large dimensions, medical staff cannot move a gamma camera around the medical institution. Its detectors have a limited field of view, which does not allow dynamic pharmacokinetic parameters to be investigated in areas of interest which are apart from each other more than $50 \mathrm{~cm}$. The above drawbacks can be overcome by using a radiometric system (RS). Such a system can employ several detectors (Pankin et al., 2018). The main advantages of this system are small size and weight enabling an examination to be carried out by placing a number of detectors in several zones of interest. Each detector can be attached to the patient's body making it possible to conduct functional tests using a RS. This approach could allow the functionality of the radionuclide diagnostics to be to significantly expanded.

The RS being developed (Fig. 1) includes a set of gamma radiation detectors (see Fig. 1, pos. 1, 2, 3, and 4). The detection units are equipped with a set of replaceable collimators (pos. 5) enabling one to effectively record RFC accumulation in zones of interest. The detector of the RS consists of CsI-Tl scintillation crystals (pos. 6) and modern small-sized silicon photoelectronic multipliers (pos. 7) or SiPM, connected with analog to digital converter (pos. 8). Each of detection units measures $3 \times 3 \times 2 \mathrm{~cm}^{3}$. Also system has a microcontroller (pos. 9) and a personal computer (pos. 10) to control measurement, data acquisition and processing, and data analysis (Pankin et al., 2018). The system under development could be particularly useful where it is essential to assess the dynamics of RFC passage, accumulation in and excretion from organs and tissues which are located at several points at some distance from each other and which would not be captured by a tomograph's field of view. Scintigraphy in the static 2D imaging mode can be carried out by step-by-step scanning using the RS where the patient cannot be delivered to a stationary installation (for example, in the surgical field). The purpose of this study is to develop a technique for diagnosing pathological processes in the liver and heart using a radiometric system and develop phantoms of the liver and single-chamber heart.

\section{Experiment and result}

Phantom tests are required to be carried out before putting any new

\footnotetext{
* Corresponding author.

E-mail address: savva.pankin@urfu.ru (S.V. Pankin).
} 

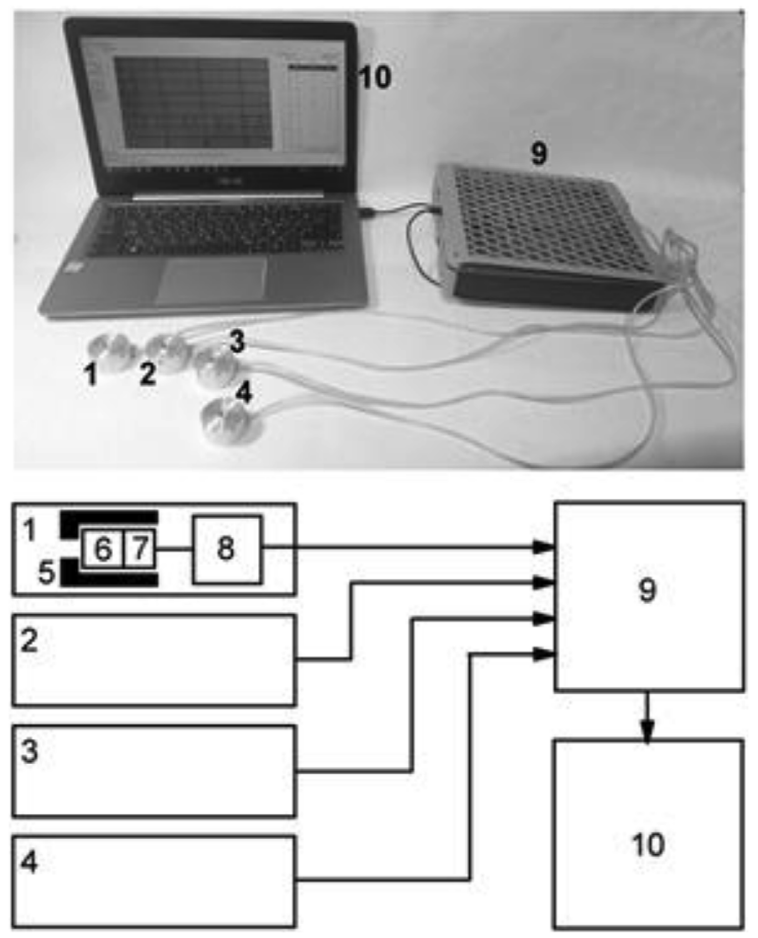

Fig. 1. General view of the radiometric system and its structure scheme.

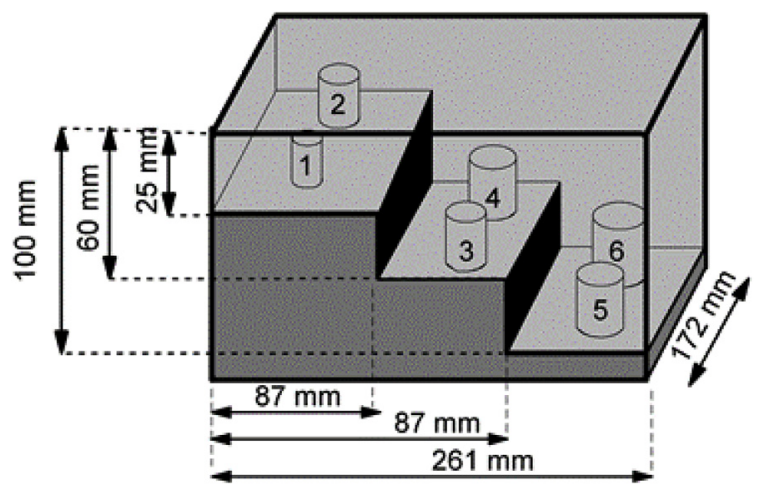

Fig. 2. Liver phantom.

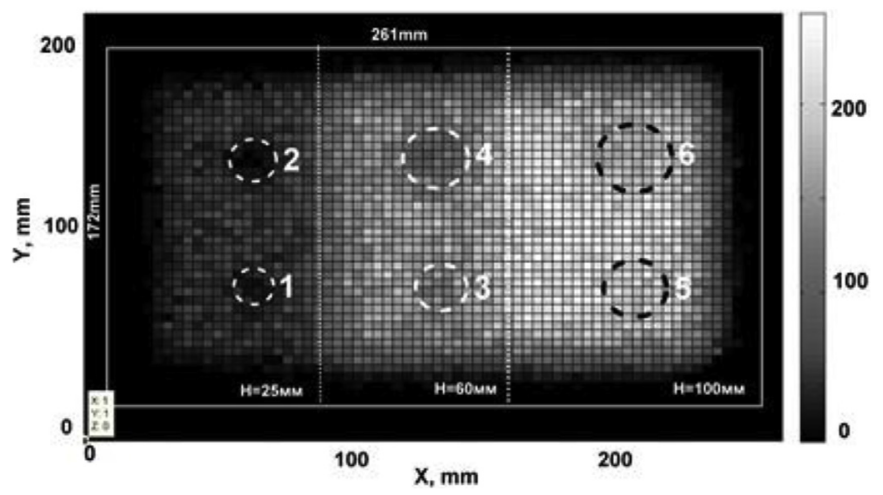

Fig. 3. Result of scanning the liver phantom with RS.

medical technique into use. The purpose of these tests is to verify the device as a whole, its configuration, ability to scan certain systems of the body, and the selection of collimation system parameters. The RS being developed has been tested using models of such biological objects as the liver and heart including mathematical descriptions to enable

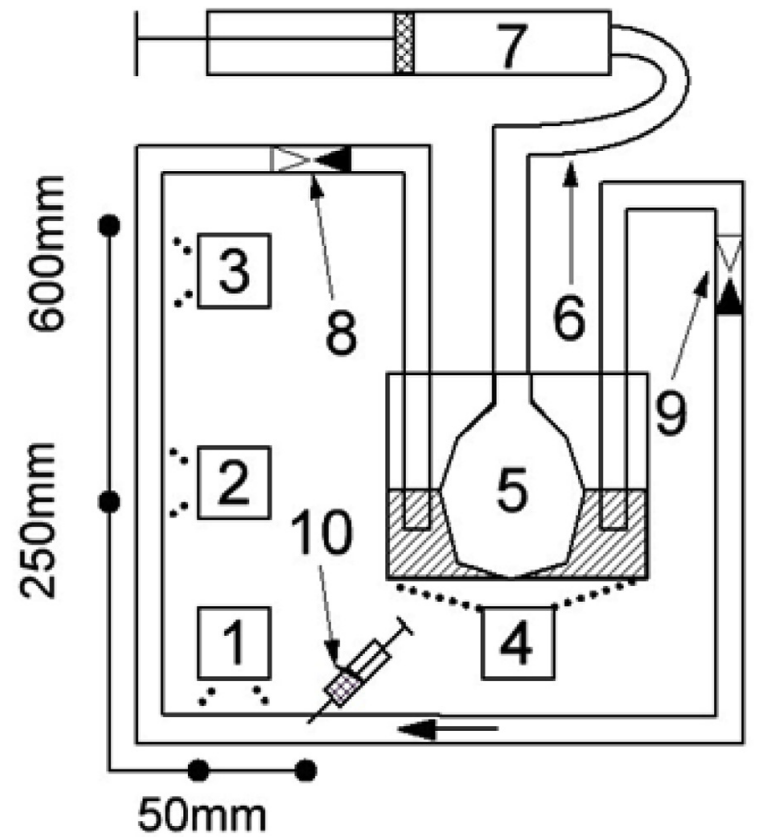

Fig. 4. General view of the single-chamber heart phantom for dynamic studies.

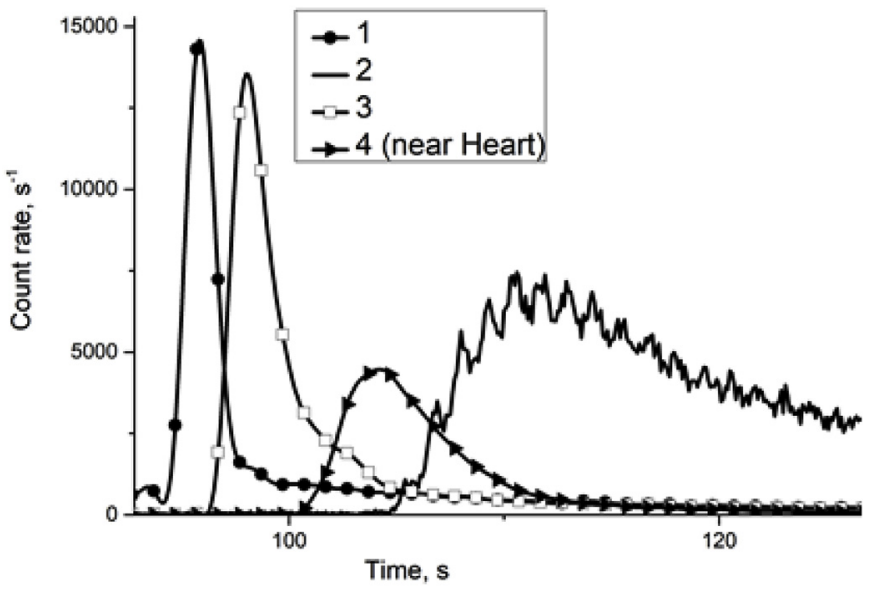

Fig. 5. Curves for changes in the activity of the first passage, measured at pos. 1 (1), pos. 2 (2), pos. 3 (3) and pos. 4 (4).

checking the key parameters of the prototype. The study was carried out at the Radionuclide Diagnosis Department of Regional Children's Clinical Hospital No. 1 in Ekaterinburg.

2.1. Static scintigraphy using the PRS in the $2 D$ scanning mode for liver pathology diagnosis as an example

To enable the RS detector to be moved in two coordinates and study RFC distribution in the patient's body, we have developed a special movement device. The experimental setup consists of a RS detector, a scanning device, RFC solution, a liver phantom. Scanning device allows 2D scanning at a minimum step of $1 \mathrm{~mm}$ (Pankin et al., 2018). Liver radionuclide diagnostics implies identifying low RFC accumulation zones which characterize the pathological process as a tumor. The main task of imaging is to assess the size, shape and position of the liver relative to the other anatomical structures and to assess the severity and nature of damage to this organ.

Medical institutions use a liver phantom (Fig. 2) for modeling liver pathologies (Zedgenidze et al., 1968). A schematic view of the phantom is shown in Fig. 2. It had three steps with two plastic cylinders placed 
on each step to simulate reduced RFC accumulation in the liver. The cylinders located on the first step measured $\varnothing 15 \times 20 \mathrm{~mm}^{3}$ and $\varnothing 20 \times 20 \mathrm{~mm}^{3}$ (see Fig. 2, pos.1, 2); on the second, $\varnothing 20 \times 20 \mathrm{~mm}^{3}$ and $\varnothing 30 \times 20 \mathrm{~mm}^{3}$ (pos.3, 4); and on the third one, $\varnothing 30 \times 20 \mathrm{~mm}^{3}$ and $\varnothing 40 \times 20 \mathrm{~mm}^{3}$ (pos.5, 6). The phantom was filled with $1.5 \mathrm{~L}$ of an RFC solution based on $\mathrm{Tc}^{99 \mathrm{~m}}$ with an activity of $27 \mathrm{MBq}$. The height of the liquid level was $25 \mathrm{~mm}$ over the first step, $60 \mathrm{~mm}$ over the second and $100 \mathrm{~mm}$ over the third. For these parameters of the phantom and a distance of $20 \mathrm{~mm}$ from the detector to the liquid surface, the resolution was $15 \mathrm{~mm}$ for the first step, $25 \mathrm{~mm}$ for the second, and $35 \mathrm{~mm}$ for the third. The technique used for calculating the collimators for the PRS and its resolving power were described in (Pankin et al., 2016).

A Z-phantom scan was obtained using the scanning unit at a scanning step of $4 \mathrm{~mm}$ and a measurement time of $1 \mathrm{~s}$ as shown in Fig. 3 "Cold areas" and the boundaries of the phantom steps are marked with white dashed lines showing the dimensions. It can be seen that for the given parameters the setup can recognize "cold areas" located on the first and second steps of the phantom. On the third step, it can distinguish only the largest "cold area". These data enable the resolving power of the 2D scanning system to be estimated. At a depth of $25 \mathrm{~mm}$, it is $20 \mathrm{~mm}$; at a depth of $60 \mathrm{~mm}, 30 \mathrm{~mm}$; and at a depth of $100 \mathrm{~mm}$, $40 \mathrm{~mm}$. Thus, the obtained resolution values are close to those calculated above.

\subsection{Dynamic scintigraphic studies using $R S$}

Tests in the dynamic mode were carried out using a prototype for the central circulation system. To this end, we developed a phantom (Fig. 4) simulating the pulmonary circuit. The phantom mimicked a pulsating single-chamber heart with one circulation circuit. The prototype used was the phantom previously developed by Pieter deBondt, MD (Pieter De Bondt, 2005). The installation consisted of 4 RS detectors (Fig. 4, pos. 1, 2, 3, 4), a single-chamber heart model (pos. 5), a circulation circuit (pos. 6), a pump (pos. 7), two check valves (pos. 8, 9) ensuring the circulation of the liquid in one direction only and a syringe for injecting the RFC (pos. 10). Using the phantom, we modeled the following simple processes: injection of RFC in various ways, blood circulation at a "cardiac pace", and dilution of the drug in the blood. Each injection contained $1 \mathrm{ml}$ of $\mathrm{Tc}^{99 \mathrm{~m}}$ solution (12 MBq activity) stained with methylene blue. The activity of the solution was monitored by an "RIS-A1" radiometer ("Amplitude" company). The RS detector collimators were made from lead and reduced the background signal. The collimators are of divergent design to ensure that the detector field of view captures just a small area of the phantom, 1-2 times larger than the dimensions of the detector. Injections were made into the tube with an insulin syringe through a needle, with three detectors located near the tube so that the distances from them to the injection site were 5, 25 and $60 \mathrm{~cm}$, respectively. The fourth detector was located at the bottom of the heart vessel so that its field of view captured the entire volume of this vessel.

Fig. 5 presents data on the pharmacokinetics obtained with the RS on the single-compartment heart phantom. The positions of the RS detectors are shown in Fig. 4 as detectors 1-4. Data processing allowed monitoring the process of bolus dilution depending on the distance of its traveling along the tube (curves 1-3) and the pulsations of the heart seen as a sawtooth change in activity (curve 4). An analysis of pharmacokinetic data helps to determine optimal distances between the RFC injection points and the positions of the detectors for the given experimental conditions, as well as blood vessel clamping options before RFC injection. Moreover, as shown in (Krotov and Pankin, 2017), this technique allows one to estimate the total volume of blood circulating in the patient's body. According to (Krotov and Pankin, 2017), it can be calculated from the area of the bolus first passage peak (on either of the curves 1,2 or 3 ), and after a certain time with the same integration parameters, from the area under the total dilution plateau. The peak area of the first bolus passage can be determined on either of the curves 1,2 or 3 , but there are limitations associated with the patient's biometric parameters and time-periodic blood flow characteristics. Placing a detector near the heart chamber vessel made it possible to determine the cardiac ejection fraction of the phantom. The tests have confirmed the possibility of using the RS for evaluating patients' hemodynamic parameters. The advantage of the system is that its detectors can be placed on patient's limbs for evaluating the dynamic parameters of the pharmacokinetics.

\section{Conclusion}

The possibilities of using the developed radiometric system for static and dynamic studies on liver and heart phantoms, respectively, have been considered. Importantly, joint use of RS and gamma camera makes it possible to supplement the time-lapse recording of pharmacokinetics in a certain part of the patient's body with information that is obtained from areas of interest outside the detector's field of view.

\section{References}

De Bondt, Pieter, Tom Claessens, Rys, Bart, De Winter, Olivier, Vandenberghe, Stijn, Patrick, Segers, Pascal, Verdonck, Andre Dierckx, Rudi, 2005. Accuracy of 4 different algorithms for the analysis of tomographic radionuclide ventriculography using a physical, dynamic 4-chamber cardiac phantom. J. Nucl. Med. 1 (46), 165-171.

Krotov, A.D., Pankin, V.V., 2017. Automated processing of first-pass radioisotope ventriculography data to determine essential central circulation parameters. AIP Conf. Proc. 1886, 020097. https://doi.org/10.1063/1.5002994.

Pankin, S.V., Sarychev, M.N., Surdo, A.I., Ivanov, V.Y., 2016. Design of collimation system for gamma probe. Izvestiya vuz. Fizika. 59 (9-2), 180-183 (In Russian). ISSN: 0021-3411.

Pankin, S.V., Sarychev, M.N., Pankin, V.V., Surdo, A.I., Krotov, A.D., Ivanov, V.Yu, Zelenin, A.V., 2018. Portable radiometric system for radionuclide diagnostics. J. ANRY. 2 (93), 40-47 (In Russian). ISSN: 2075-1338.

Rosenthal, M.S., Cullom, J., Hawkins, W., Moore, S.C., Tsui, B.M.W., Yester, M., 1995. Quantitative SPECT imaging: a review and recommendations by the focus committee of the society of nuclear medicine computer and instrumentation council. J NuciMed 36 (8), 1489-1513.

Zedgenidze, G.A., Zubovskiy, G.A., 1968. Izucheniye Gemodinamiki S Pomoshyu Radioaktivnyh Izotopov. Klinicheskaya Radioizotopnaya Diagnostika, Moscow (In Russian). 